\title{
Le financement corporatif de la recherche universitaire
}

\author{
MICHEL LECLERC*
}

\section{RÉSUMÉ}

L'évaluation du niveau du financement privé de la recherche universitaire met en cause des sources statistiques incompatibles mais tout autant l'ambiguité même de l'objet dans la mesure où ces statistiques incluent un financement parapublic. Les statistiques sur le financement privé regroupent indifféremment les revenus d'entreprises et les revenus de sociétés d'État; à l'inverse, celles sur le financement corporatif les distinguent.

Les données relatives au Québec permettent d'observer que le déclin du financement corporatif est imputable non pas au secteur privé, dont le niveau de dépenses est demeuré pratiquement stationnaire entre 1980 et 1985, mais aux secteurs publics et parapublics, dont l'aide financière, en proportion du financement corporatif global, a chuté de moitié au cours de cette période. On constate aussi que la structure du financement corporatif épouse la division linguistique de l'enseignement supérieur. Ainsi les universités francophones dépendent-elles davantage des fonds versés par les sociétés d'État que leurs consoeurs anglophones. L'intensité des liaisons université-entreprise, telle que mesurée par les indicateurs de performance, est fortement conditionnée par la différenciation linguistique.

\section{SUMMARY}

Assessment of private funding for university research reveals that the various sources of statistical data on which such evaluation is too often based are contradictory, and that a great deal of ambiguity exists as to what to include under the subject itself, e.g. whether funding from Crown corporations is private or public. Statistics for private funding make no distinction between private corporate funding and funding from Crown corporations; however, statistics for corporate funding consider them separately.

Data for Québec show that the drop in corporate funding is not attributable to the private sector - whose expenditures have remained virtually unchanged from 1980 to 1985 - but rather to the public and parapublic sectors, whose financial 
assistance fell by nearly 50\% over the same period in comparison to overall corporate funding. Moreover, it was noted that the corporate funding structure mirrors the linguistic division of higher education. French-language universities are more dependent on funding from Crown corporations than are their English-language counterparts. The closeness of university-corporation ties, as measured by performance indicator, is to a great extent conditioned by linguistic differences.

Tant et aussi longtemps que l'université n'a incarné que la quête désintéressée du savoir, le phénomène des relations universitê-entreprise n'a suscité qu'un intérêt mitigé.

Mais, d'une part, sous l'effet des restrictions financières imposées depuis quelques années à l'enseignement supérieur et en particulier à la recherche universitaire, les universités se sont vu soudainement contraintes, dans la majorité des pays, à chercher des sources de financement additionnelles. D'autre part, s'est accru l'espoir ou le désir que l'université participe davantage au développement social comme au développement industriel et en retire des avantages financiers.

Presque partout le diagnostic est le même: la part des dépenses de la recherche universitaire financée à même le budget général des universités est en diminution constante, tandis que la part des activités de recherche soutenues grâce au financement corporatif tend à s'accroître.

Face à cette pression financière, la conception traditionnelle essentiellement humaniste de l'université a dû céder la place à une conception nettement plus utilitariste de sa mission et de ses objectifs.

Selon un récent rapport de l'OCDE (1987b, p. 19), "les pouvoirs publics et la population demandent de plus en plus aux universités de contribuer, par leurs fonctions de recherche, d'enseignement et de formation, à renforcer la compétitivité de l'économie. On attend des universités qu'elles favorisent l'innovation, qu'elles montrent comment appliquer les résultats de la recherche et en tirer utilement profit, qu'elles indiquent les domaines vers lesquels il faut diriger les ressources pour pouvoir améliorer la productivité et les résultats économiques".

La volonté des pouvoirs publics d'inciter à l'utilisation des connaissances affecte les universités à plus d'un titre: elle modifie la fonction et les objectifs de l'université; elle modifie les règles d'organisation de la recherche; elle entraîne une augmentation des clientèles étudiantes dans les filières à caractère professionnel plus orientées vers la "production de richesse"; elle favorise un resserrement des liens entre l'université et l'entreprise, entre les technologies de pointe et la recherche fondamentale, entre la science et la technologie. Nombre d'universitaires s'inquiètent que l'on détourne ainsi la fonction recherche vers des travaux de recherche appliquée dont le financement implique des contraintes parfois incompatibles avec l'éthique universitaire. 
Il est vrai que les postulats relatifs à l'accroissement de l'efficacité de la recherche universitaire, sur lesquels reposent les mesures de rapprochement université-industrie, ont trop tendance à se référer à un principe exclusif et à céder à l'instance du besoin immédiat. Par ailleurs, il n'est pas moins certain que "l'université ne pourra pas fournir sa contribution la plus grande à la prospérité de la société si elle se conforme étroitement aux impératifs du moment" (Blume, 1984, p. 195).

Mais quelles limites raisonnables fixer au financement corporatif au nom de l'efficacité à long terme de la recherche universitaire? La valeur du raisonnable ne saurait se trouver dans la seule quantification, mais "il est clair qu'elle est dépassée lorsque l'université perd sa liberté de choix des sujets et des méthodes de recherche" (Philippart, 1985, p. 209).

Ce risque d'un asservissement de la recherche universitaire à l'entreprise privée a été maintes fois évoqué (Dickson, 1984; Ruscio, 1984; Ashworth, 1985) mais force est d'admettre qu'il n'est qu'exceptionnellement corroboré par les faits. Est-il même certain que la contribution directe des entreprises aux dépenses de recherche des universités du Québec soit actuellement supérieure à son niveau de 1980? L'entreprise reconnait-elle à la question des collaborations universitéentreprise le même caractère prioritaire que lui confère le discours gouvernemental?

C'est précisément à ces questions que nous allons maintenant tenter d'apporter des réponses. A cette fin, nous évaluerons l'importance relative des contributions "directes" des entreprises aux activités de recherché universitaire. Nous examinerons également quelles variations annuelles ont affecté l'intensité du financement industriel entre 1980 et 1985.

\section{Financement privé et financement corporatif}

En ce qui a trait au financement privé de la recherche universitaire, il est d'usage de confondre les revenus provenant d'entreprises commerciales de ceux qui émanent des sociétés d'État ou de corporations publiques. On s'empêche ainsi et voir qu'une fraction importante des revenus issus de ce qu'on appelle communément le financement "privé" ressortit en réalité aux institutions publiques et parapubliques à vocation industrielle ou commerciale.

Dans le présent article, le financement "privé" sera considéré comme une composante distincte du financement corporatif. En effet, le financement corporatif ne constitue pas une entité homogène. En l'occurrence, il est apparu plus pertinent de distinguer le financement corporatif privé, imputable à l'entreprise privée stricto sensu, du financement corporatif public ou parapublic, qui relève de l'État par l'entremise des corporations ou des sociétés dont il a la charge. Par ailleurs, le financement corporatif désigne exclusivement les fonds provenant de "compagnies", de "sociétés" ou de "corporations" (privées, publiques ou parapubliques), à l'exclusion des revenus issus d'associations, de particuliers ou, plus généralement, d'institutions sans but lucratif (ISBL).

Le niveau de financement s'en trouve ainsi fortement réduit mais une telle 
évaluation fournit une mesure du financement corporatif de la recherche universitaire beaucoup plus conforme à la réalité. En effet, de façon générale, seuls les revenus issus des "compagnies" et des "corporations" sont réellement destinés à supporter l'effort de R-D universitaire, les ressources obtenues des "fondations" ou des "associations" servant essentiellement à soutenir la mission éducative et culturelle de l'université.

\section{Déclin et croissance du financement privé: comparaisons internationales}

Quel que soit le pays considéré, le financement privé ne constitue qu'une source de fonds marginale pour la recherche universitaire. La confrontation des statistiques nationales (tableau 1) indique qu'en 1984, aux États-Unis, le financement privé ne représentait guère que $3,5 \%$ des budgets totaux de la recherche universitaire; au Japon, ce type de financement équivalait à seulement $2,3 \%$, tandis qu'en R.F.A., selon des données de 1983 , il correspondait à 5,3\% des dépenses totales de la RDU (recherche et développement universitaire). Avec un niveau de financement privé égal à $3,2 \%$, le Canada se situait donc parmi les pays où les liaisons entrepriseuniversité semblaient les plus développées, tout au moins du strict point de vue du financement direct.

Le niveau du financement privé n'est cependant nulle part si élevé qu'il puisse engendrer une dépendance réelle de l'université à l'égard de finalités dictées par l'industrie ou encore qu'il en vienne bientôt à se substituer, fut-ce partiellement, au financement public. En effet, "la collaboration université-entreprise ne génère pas", selon la conclusion d'une étude du Conseil de la Science et de la Technologie (1986, p. 19), “... des revenus assez significatifs pour qu'éventuellement, les gouvernements puissent compter sur les entreprises pour résoudre les problèmes de financement de la recherche universitaire". Aussi bien la volonté d'allégement des finances publiques ne constitue-t-elle pas une explication suffisante du renforcement de la collaboration entre l'université et l'entreprise (Advisory Board for the Research Councils, 1984, p. 7). Paradoxalement, la détérioration du soutien gouvernemental, qui a obligé les universités à se tourner vers des sources nouvelles de financement, apparaît souvent comme la raison initiale du développement de cette collaboration (Kennedy, 1986, p. 265).

Le financement corporatif ne représente donc pas une solution adéquate à la crise financière que traversent actuellement les universités, d'autant plus que ce type de financement est soumis à des variations annuelles importantes (tableau 2) qui rendent aléatoire toute tentative de planification financière de la recherche. En somme, “à ce niveau, le problème n'est pas l'augmentation du financement industriel, mais plutôt dans plusieurs pays la diminution du financement public" (OCDE, 1987a, p. 144).

\section{La sous-estimation du financement corporatif de la R-D universitaire}

Les statistiques sur les modes de financement université-entreprise, fondées sur les 
Tableau 1: Part de la RDU financée par le secteur privé dans certains pays de 1 'OCOE, 1980-1984 (SNG/SSH)*

\begin{tabular}{|c|c|c|c|c|c|}
\hline & 1980 & 1981 & 1982 & 1983 & 1984 \\
\hline États-Unis & 2,5 & 2,7 & 3,0 & 3,2 & 3,5 \\
\hline Japon & 1,4 & 1,5 & 1,6 & 1,8 & 2,3 \\
\hline R.F.A. & $2,1^{1}$ & 1,9 & --- & 5,3 & --- \\
\hline France & $2,7^{1}$ & 1,3 & 1,3 & 1,3 & 1,5 \\
\hline Royaume-Uni & --- & 2,8 & --- & 3,1 & --- \\
\hline Canada & 0,3 & 3,1 & 2,3 & 2,4 & 3,2 \\
\hline Pays-Bas & -- & 0,3 & 0,5 & 0,6 & 0,9 \\
\hline Italie & 1,3 & 2,7 & 2,3 & 0,5 & -- \\
\hline
\end{tabular}

11979

*SNG: Sciences naturelles et du génie

SSH: Sciences sociales et humaines

Sources: OCDE, Indicateurs OCDE de la Science et de la Technologie 3, SPT (87)9, Annexe, Tableau 46 . 
Tableau 2: Dépenses de la R-D universitaire financées par les entreprises (z)1, Québec, Ontario, Canada, 1980-1985 (SNG)

\begin{tabular}{lcccccc} 
& 1980 & 1981 & 1982 & 1983 & 1984 & 1985 \\
\hline Québec & 5,9 & 5,8 & 3,4 & 3,7 & 3,4 & $3,5(3,2)^{\star}$ \\
Ontario & 3,5 & 3,5 & 3,0 & 2,9 & 4,5 & $6,1(5,3)^{\star}$ \\
Canada & 3,5 & 3,5 & 2,6 & 2,7 & 3,5 & $4,4(4,0)^{\star}$ \\
\hline
\end{tabular}

1 Statistique Canada inclut les entreprises publiques et parapubliques dans la catégorie "entreprises".

* Les chiffres entre parenthèses incluent la contribution des SSH.

Sources: Statistique Canada (1987). Les estimations des dépenses canadiennes au titre de la recherche et du développement par région, 1979-1985, Ottawa; Séries spéciales fournies par Statistique Canada. 
dépenses estimées de R-D, ont tendance à sous-estimer le niveau de financement réellement assumé par les corporations publiques ou privées et à tracer de surcroît un portrait fort incomplet des nouvelles formes de relations financières qui se sont nouées entre elles.

Les statistiques nationales ne comptabilisent que les contributions directes aux universités. Elles ne mesurent, par conséquent, qu'un certain type d'échanges économiques de nature plus traditionnelle. Ainsi, tous les échanges ou accords université-entreprise qui n'impliquent pas un financement formel (prêt ou don d'équipement, échange de personnel, formation de main-d'oeuvre, etc) n'apparaissent pas dans les statistiques officielles indépendamment de leur importance stratégique réelle ou de leur valeur économique indirecte dans l'ensemble des relations entreprise-université.

"En fait, comme le soulignait un rapport de l'OCDE (1984, p. 16), il se pourrait qu'au total ces échanges et ces relations jouent un rôle beaucoup plus crucial à l'heure actuelle que le financement effectif par l'industrie de recherche dans les universités."

En réalité, la contribution réelle des entreprises privées ou publiques au financement de la R-D universitaire pourrait être au moins le double, sinon plus, de celle qui est officiellement enregistrée par les indicateurs disponibles (OCDE, 1987a, p. 143).

\section{La situation du financement corporatif de la RDU au Canada: effondrement de l'aide financière au Québec et renversement de la position des provinces depuis 1980}

En 1985, les entreprises supportaient financièrement 4,4\% de la R-D universitaire réalisée au Canada dans le domaine des sciences naturelles et du génie (tableau 2). Au Québec, la contribution des entreprises atteignait 3,5\%, alors qu'en Ontario elle s'élevait à $6,1 \%$. Sauf au Québec, où elle a diminué, la part du financement corporatif dans l'ensemble des dépenses totales de la R-D universitaire a augmenté partout au Canada entre 1980 et 1985.

La récession économique de 1982 a entraîné un déclin soudain du niveau de soutien industriel partout au Canada et a marqué du même coup la fin de la prééminence du Québec en matière de financement industriel à la RDU. En effet, contrairement à ce qui peut être observé dans les autres régions canadiennes, la collaboration université-entreprise au Québec n'a jamais surmonté le choc de la crise économique.

Alors qu'en 1980 le financement corporatif au Québec totalisait 5,9\% des dépenses de la R-D universitaire (SNG), en 1985 cette part relative était réduite de $40,6 \%$ et n'équivalait plus qu'à $3,5 \%$ de la RDU. En revanche, au cours de la même période la part du financement corporatif augmentait de $74,5 \%$ en terme relatif en Ontario (passant de $3,5 \%$ à $6,1 \%$ ) et de $25,7 \%$ dans l'ensemble des provinces canadiennes (cette part grimpant de 3,5\% à 4,4\% de la RDU totale). En somme, les positions des provinces en 1985 sont l'inverse complètement de ce 
qu'elles étaient en 1980, vu la diminution constante des fonds publics versés à la recherche universitaire.

\section{La situation du financement industriel de la RDU au Québec: diminution relative du support corporatif public et parapublic}

En comparaison des données estimatives recueillies par Statistique Canada, les statistiques sur la recherche subventionnée et commanditée compilées par le ministère de l'Enseignement supérieur et de la Science comportent deux avantages importants: 1. elles enregistrent les revenus comptabilisés de la recherche universitaire; 2 . elles distinguent les revenus de source corporative selon leur origine: entreprises privées, entreprises publiques ou parapubliques.

Par conséquent, ces données permettent de dresser un bilan beaucoup plus fidèle et détaillé du soutien des corporations à la recherche universitaire. Les résultats de cette enquête (tableau 3) mettent en évidence les deux caractéristiques suivantes: - la participation financière des corporations privées et publiques aux activités de la R-D universitaire au Québec est supérieure à ce que laissait entrevoir l'évaluation des dépenses estimées de la R-D. En fait, l'enquête du MESS révèle que le financement corporatif (privé et public) de la R-D universitaire au Québec est supérieur de 33,3\%, en termes relatifs, à l'estimation de Statistique Canada et correspond, par conséquent, à $4,8 \%$ plutôt qu'à $3,2 \%$ des dépenses totales de la recherche universitaire;

- la part du financement privé dans l'effort global du financement corporatif (privé et public) tend à s'accroître au cours des années, celle-ci passant de $63 \%$ du financement corporatif total en $1980(3,9 \% / 6,2 \%)$ à $77 \%(3,7 \% / 4,8 \%)$ en 1985 . Bref, depuis 1980, le fardeau du financement industriel de la R-D universitaire est de plus en plus à la charge des entreprises privées et incombe de moins en moins aux entreprises publiques et parapubliques, qui n'assument plus que le tiers du financement corporatif de la RDU.

\section{Les universités et le contexte socio-économique du financement industriel de la RDU}

Le financement corporatif n'est pas uniformément réparti entre les universités québécoises. Une série de facteurs socio-économiques influencent la capacité d'une université d'accaparer les revenus de source corporative: la qualité et la productivité de ses chercheurs, sa proximité géographique des grandes zones urbaines, les particularités de l'environnement industriel, ses liens historiques et socio-culturels avec la communauté industrielle, etc.

On sait par ailleurs que ce sont les grandes entreprises plutôt que les PME qui financent la R-D universitaire (Lemasson, 1985) et que les attentes d'une industrie locale à l'endroit d'une université sont de nature fort différente de celles manifestées par une industrie nationale ou transnationale. Pour les innovations majeures, par exemple, les industries transnationales ont surtout besoin de travaux 
Tableau 3: Variations annuelles de la part des revenus de source corporative (privée et publique) en $q$ des revenus totaux de la RDU au Québec, 1980-1985

$\begin{array}{cccccc}1980 & 1981 & 1982 & 1983 & 1984 & 1985 \\ \% & \% & \% & \% & \% & \%\end{array}$

Recherche subventionnée

(Toutes disciplines)

- Corporations privées

6,2

6,4

4,8

5,1

4,6

4,8

- Corporations privées

3,9

4,9

3,4

3,7

3,3

3,7

Revenus estimés de la $R-D$

(Sciences naturelles

et du génie seulement)

$5,9 \quad 5,8 \quad 3,4 \quad 3,3^{\star} \quad 3,3^{\star} \quad 3,2^{\star}$

* Inclut les revenus des SNG et des SSH.

Sources: Statistique Canada (1986). Estimation des dépenses au titre de la recherche et du développement dans le secteur de l'enseignement supérieur, 1984-1985, Ottawa; Statistique Canada (1985). Les estimations des depenses canadiennes au titre de la recherche et du développement, par region, 1980-1983, Ottawa; Statistique Canada (1985). Estimation des dépenses au titre de la recherche et du développement dans le secteur de T'enseignement superieur, 1983-1984, Ottawa; Statistique Canada (I987). Les estimations des depenses canadiennes au titre de la recherche et du développement, par région, 1979 a 1985 , ottawa; O.G.E.R.U. La recherche subventionnée et commanditèe dans les universités du Québec, Rapport statístique 1980-1981 à 1982-1983, Quebec. Pour Tes donnees 1983-1984 et 1984-1985 les résultats sont tírés de 1 'analyse de transcriptions informatiques non publiées ( tableaux T/07-0-SC-1, T/07-0-SC-3, T/07-0-SC-4) fournies par la D.G.E.R.U.. 
de recherche fondamentale menés dans des institutions prestigieuses (les "distinguished universities") plutôt que de travaux appliqués de recherche-développement réalisés localement (Blume, 1985).

Les universités québécoises sont bien sûr très diversement affectées par ces contraintes environnementales mais elles font toutes face au même désintérêt des PME à l'égard de la concertation université-entreprise. Qu'il suffise simplement de rappeler qu'à l'occasion du premier Sommet des industries de haute technologie du Québec, tenu le 21 septembre 1987, les représentants des PME québécoises ont exprimé leur refus de s'engager dans des activités de recherche impliquant les universités comme partenaires (Chapdelaine, 1987). À défaut de pouvoir étudier en détail le rôle de chacun des facteurs environnementaux déterminant l'incidence des liaisons université-entreprise, on peut au moins analyser la structure du financement corporatif dans les universités québécoises. Cet examen, on le verra, permet de caractériser la situation des universités en matière de financement de la R-D à partir de quatre conclusions principales: 1. l'instabilité du financement corporatif; 2 . la forte prédominance des revenus de source corporative privée; 3 . l'existence d'écarts significatifs entre les universités francophones et anglophones quant à l'origine des fonds corporatifs; 4. l'inégalité des performances universitaires pour l'obtention des revenus de source corporative privée et publique.

Examinons donc plus en détail chacune de ces conclusions.

\section{L'INSTABILITÉ DU FINANCEMENT CORPORATIF}

Si la répartition globale des revenus corporatifs alloués au réseau universitaire entre 1981 et 1985 a peu affecté la part relative accaparée par les sous-réseaux francophone $(68,0 \%)$ et anglophone $(35,0 \%)$, en revanche la distribution des revenus de source corporative entre les universités a beaucoup fluctué (tableau 4). Ainsi, au cours de cette période, l'Université Laval et l'Université du Québec ont vu doubler leur part des revenus totaux de source corporative, ceux-ci passant graduellement de $7 \%$ à 14\%. Dans le cas de l'Université Laval, cependant, les variations annuelles ont été plus importantes qu'à l'Université du Québec. La part des revenus totaux de source corporative accaparés par l'Université de Montréal en 1985 correspond à sa part de 1981 (environ 26\%) mais celle-ci a beaucoup oscillé au cours des années, passant successivement de 22\% (1982) à 26\% (1983) et de $26 \%$ à $34 \%$ (1984). Les données relatives à l'Université de Sherbrooke demeurent encore incomplètes et ne permettent donc pas de conclure. Dans le réseau des universités anglophones, la concentration des revenus de source corporative au sein de l'Université McGill reste constante au cours des années, en dépit d'un léger déclin $(-3,7 \%)$ qui, pour l'essentiel, semble profiter surtout à l'Université Concordia, dont la part relative augmente de $1,4 \%$ en 1981 à $3,6 \%$ en 1985.

On l'aura peut-être compris le caractère contingent du financement corporatif de la RDU se concilie difficilement avec les règles institutionnelles de la planification budgétaire. Le financement industriel, on l'a constaté plus tôt, obéit trop étroitement aux aléas de la conjoncture économique pour qu'il soit considéré 
Taleau 4: Financenent corporatff privé et publtc de la recherche universtentre w Qübec,
$1981-1982$ a $1995-1986$

\begin{tabular}{|c|c|c|c|c|c|c|c|c|c|c|c|c|c|c|c|}
\hline & \multicolumn{5}{|c|}{$\begin{array}{l}\text { Revenus de source corporattive } \\
\text { privée et publlque en } \$ \text { des } \\
\text { revenus totaux de la recherche }\end{array}$} & \multicolumn{5}{|c|}{$\begin{array}{l}\text { Revenus de source corporative } \\
\text { privée en des revenus totaux de } \\
\text { la recherche }\end{array}$} & \multicolumn{5}{|c|}{$\begin{array}{l}\text { Parts des revenus totaux de source } \\
\text { corporative du réseau }\end{array}$} \\
\hline & 1981 & 1982 & 1983 & 1984 & 1985 & 1981 & 1982 & 1983 & 1984 & 1985 & 1981 & 1982 & 1983 & 1984 & 1985 \\
\hline Universités francophones & 6,3 & 4,2 & 5,1 & 4,7 & 4,6 & 4,2 & 2,8 & 3,1 & 2.7 & 3,2 & 64.0 & 65,3 & 68,5 & 63,6 & 65.3 \\
\hline Laval & 2,5 & 1,8 & 1,3 & 3,6 & 3,6 & 1,3 & 1,4 & 1,0 & 3,0 & 3,2 & 7,5 & 7,0 & 5,0 & 16,2 & 14,6 \\
\hline Montréal 1 & 7,0 & 4,6 & 5,4 & 6,1 & 5,5 & 4,7 & 1,7 & 2,0 & 2,5 & 2,8 & 26,2 & 22,0 & 26,9 & 34,3 & 26,9 \\
\hline Sherbrooke & 16,8 & 11,5 & 9.7 & N.D. & $5.6^{*}$ & 13,3 & 9,8 & 8,8 & N.O. & $4,2^{\star}$ & 22,5 & 23.0 & 19,3 & N.D. & $9,7^{*}$ \\
\hline U. 0. & 3.8 & 4,7 & 6,3 & 3,8 & 4,2 & 1,7 & 1.4 & 4,1 & 2,6 & 3,2 & 7,8 & 13,3 & 17,2 & 13,1 & 14,0 \\
\hline Universités anglophones & 6,5 & 4,7 & 5,0 & 4,5 & 5,2 & 6,1 & 4,6 & 4,8 & 4.4 & 4,8 & 36,0 & 34.7 & 31,5 & 36,4 & $34,6^{\circ}$ \\
\hline Bishops & $\cdots$ & $-\cdot$ & $\ldots$ & $\ldots$ & -- & - & - & $\ldots$ & $\ldots$ & $\ldots$ & $\ldots$ & $\ldots$ & $\ldots$ & - & $\ldots$ \\
\hline$M C G(11$ & 7,3 & 4,9 & 5,3 & 4,6 & 5,3 & 6,9 & 4,8 & 5,0 & 4,4 & 4,9 & 34,6 & 31,5 & 29,0 & 32,0 & 30,9 \\
\hline Concordia & 1,7 & 3,5 & 3,0 & 4,4 & 4,2 & 1,7 & 3,1 & 3,0 & 4,4 & 4,2 & 1,4 & 3,2 & 2,3 & 4,4 & 3,6 \\
\hline Ensemble des universitës & 6,4 & 4.8 & 5,1 & 4,6 & 4,8 & 4.9 & 3,4 & 3,7 & 3,3 & 3.7 & 100,0 & 100,0 & 100.0 & 100.0 & 100,0 \\
\hline
\end{tabular}

1 Université de Montréal - HEC - Polytechnique.

* Données provisotres.

Sources: Données informatiques non publiées fourntes par la D.G.E.R.U.. 
comme un élément fiable de prévision budgétaire en matière de recherche. Dans le contexte actuel, le financement corporatif apparaît essentiellement comme un revenu d'appoint de la recherche universitaire dont les justifications ressortissent avant tout aux critères économiques.

\section{LA FORTE PRÉDOMINANCE DES REVENUS DE SOURCE CORPORATIVE PRIVEE}

Le financement corporatif de la R-D universitaire est essentiellement constitué de capitaux issus d'entreprises privées, mais les revenus d'entreprises publiques ou parapubliques supportent encore près de $25 \%$ du financement corporatif global. En 1981, les revenus de corporations privées représentaient 76,5\% du financement total de source corporative et finançaient 4,9\% des activités de R-D universitaire au Québec; en 1985, ces revenus représentaient plus de $77 \%$ du financement privé global et supportaient 3,7\% de l'effort de R-D universitaire. À l'inverse, entre 1980 et 1985 l'aide financière des entreprises publiques et parapubliques en proportion du financement corporatif global chutait de moitié, passant de $2,3 \%$ des revenus totaux de la R-D universitaire à $1,1 \%$ seulement.

Il est dans l'ordre des choses que le financement corporatif de la recherche universitaire incombe prioritairement à l'entreprise privée. Peut-être la tendance à diminuer du financement parapublic des sociétés d'État reflète-t-elle une volonté gouvernementale de limiter ses initiatives et de susciter par tous les moyens une contribution accrue de l'entreprise privée?

\section{DES ECARTS SIGNIFICATIFS ENTRE LES UNIVERSITÉS FRANCOPHONES ET} ANGLOPHONES QUANT À L'ORIGINE DES FONDS CORPORATIFS

La structure du financement corporatif varie selon les caractéristiques linguistiques des universités. Les universités francophones dépendent davantage des fonds versés par des sociétés d'État, des corporations publiques ou parapubliques que leurs consoeurs anglophones. En 1985, par exemple, 69,5\% des revenus de source corporative versés aux universités francophones provenaient de l'industrie privée, comparativement à $92,3 \%$ dans le cas des universités anglophones. À l'Université de Montréal, où le financement provenant des corporations équivaut à $5,5 \%$ des revenus totaux de la recherche, la dépendance à l'égard des revenus de source corporative publique représente un peu plus de $50 \%$ du financement corporatif total. C'est d'ailleurs l'Université de Montréal qui se révèle la moins apte parmi l'ensemble des universités québécoises à attirer vers elle des revenus de recherche de sources industrielles privées, puisque ceux-ci ne totalisaient en 1985 que 2,8\% des revenus globaux de la recherche. Aux universités de Sherbrooke et du Québec, la proportion des revenus d'origine privée atteint $75 \%$, tandis qu'à l'Université Laval cette proportion se situe à $88,8 \%$ des revenus totaux de source corporative, soit à un niveau légèrement inférieur à celui de l'Université McGill $(92,3 \%)$. Si l'Université Laval dépend si peu des revenus corporatifs de source publique, c'est essentiellement en raison de la répartition géographique des sociétés d'État, les plus importantes sociétés publiques sur le plan des activités de R-D (Hydro-Quebec, Société nationale de l'amiante; Nouveler, Société des 
loteries et courses) ayant leur siège social hors de la région de Québec. Selon une récente étude du Conseil de la Science et de la Technologie (1987, p. 105), la région de Québec ne regroupe qu'une très faible partie des activités de R-D des sociétés d'État, celles-ci correspondant à seulement $0,8 \%$ de leurs dépenses intra-muros totales de R-D.

En somme, l'aptitude des universités francophones à obtenir des fonds corporatifs dépend encore largement $(+$ de $30 \%$ ) du support des corporations publiques et parapubliques. Sans ce soutien, la part du financement industriel accaparée par les universités francophones ne totaliserait plus que 3,2\% de leurs revenus totaux de recherche, comparativement à $4,8 \%$ pour les universités anglophones, cette performance situant d'emblée ces dernières au sommet des pays industrialisés quant à l'intensité des relations université-entreprise, toute discussion sur la compatibilité des indicateurs internationaux mise à part.

\section{L'INEGALITE DES PERFORMANCES UNIVERSITAIRES POUR L'OBTENTION DES} REVENUS DE SOURCE CORPORATIVE PRIVEE ET PUBLIQUE

Si nous voulons réaliser une mesure de l'intensité réelle des liaisons universitéentreprise, il faut recourir à un indice de performance ${ }^{2}$ qui corrige les distorsions dues à la taille des populations de chercheurs. À la lumière des données du tableau 5 , la performance globale des universités francophones et anglophones apparaît plus ou moins satisfaisante, selon que l'on considère les revenus corporatifs comme des revenus indifférenciés ou selon, au contraire, que l'on établit une distinction statistique entre les revenus de source corporative privée et publique. À cet égard, les données indiquent, encore une fois, que l'apport des revenus de source corporative publique contribue à améliorer significativement la performance globale des universités francophones, à l'exception de l'université de Sherbrooke dont le modèle de performance correspond davantage à ceux des universités anglophones, où les revenus de source corporative publique ont pour effet de diminuer la performance globale.

Ainsi, en 1983-1984, la performance globale des universités francophones correspondait à un indice de 1,01 , tandis que celle des universités anglophones $(0,97)$ indiquait que celles-ci accaparaient une part des revenus globaux de source corporative inférieure à ce que laissait présumer la taille relative de leur population de professeurs-chercheurs. Avec à peine plus de $7 \%$ du personnel enseignant régulier, l'Université de Sherbrooke attirait vers elle 19,3\% des revenus totaux de source corporative et $24,2 \%$ des revenus d'entreprises privées. Cette performance était près de deux fois supérieure à celle enregistrée par l'Université McGill $(1,38$ et 1,81$)$. La performance de l'Université Laval, qui apparaît médiocre quel que soit l'indice utilisé $(0,36$ et 0,39$)$, doit rester l'objet d'un jugement fort nuancé tant il s'agit d'un cas d'exception. Jusqu'en 1983, en effet, cette université s'est très peu préoccupée d'établir des liaisons financières solides avec l'entreprise. À partir de 1984, cependant, l'Université Laval a amorcé un rattrapage vigoureux et triplait soudainement sa part des revenus industriels, qui atteignait cette année-là 16,2\% des revenus totaux de source corporative versés aux universités québécoises. En 
de performance des universitēs québécoises, 1983-1984

\begin{tabular}{|c|c|c|c|c|}
\hline \multirow{2}{*}{$\begin{array}{l}\% \text { des revenus } \\
\text { totaux de source } \\
\text { corporative/ } \\
\text { réseau } \\
\text { (1) }\end{array}$} & \multirow{2}{*}{$\begin{array}{l}\text { \% des revenus de } \\
\text { source corpora- } \\
\text { tive priveel } \\
\text { réseau } \\
\text { (2) }\end{array}$} & \multirow{2}{*}{$\begin{array}{l}\text { \% des profs. } \\
\text { à plein temps/ } \\
\text { rêseau } \\
\text { (3) }\end{array}$} & \multicolumn{2}{|c|}{$\begin{array}{l}\text { Indices de } \\
\text { performance }\end{array}$} \\
\hline & & & $(1) /(3)$ & $(2) /(3)$ \\
\hline 68,5 & 58,7 & 67,6 & 1,01 & 0,87 \\
\hline $\begin{array}{r}5,0 \\
26,9 \\
19,3 \\
17,2\end{array}$ & $\begin{array}{r}5,4 \\
13,7 \\
24,2 \\
15,4\end{array}$ & $\begin{array}{r}13,7 \\
23,7 \\
7,5 \\
22,7\end{array}$ & $\begin{array}{l}0,36 \\
1,13 \\
2,57 \\
0,76\end{array}$ & $\begin{array}{l}0,39 \\
0,58 \\
3,23 \\
0,68\end{array}$ \\
\hline 31,5 & 41,3 & 32,4 & 0,97 & 1,27 \\
\hline $\begin{array}{r}0,0 \\
29,0 \\
2,3\end{array}$ & $\begin{array}{r}0,0 \\
38,1 \\
3,2\end{array}$ & $\begin{array}{r}1,1 \\
21,0 \\
10,3\end{array}$ & $\begin{array}{l}0,00 \\
1,38 \\
0,22\end{array}$ & $\begin{array}{l}0,00 \\
1,81 \\
0,31\end{array}$ \\
\hline
\end{tabular}

Sources: Données ińformatiques non publiẻes fournies par la D.G.E.R.U.; Association des universités et des collèges du Canada (1984). Compendium de statistiques concernant les universités, édition 1984, 0ttawa. 
conséquence, contrairement aux autres universités où la progression relative des revenus corporatifs a été plus régulière depuis 1983 , les indices de performance relatifs à l'Université Laval ne correspondent plus aussi fidèlement à la situation présente. Compte tenu de la stabilité actuelle des effectifs professoraux, on peut donc estimer que depuis 1984 la performance globale de l'Université Laval a été supérieure à l'unité.

À l'Université de Montréal, les revenus corporatifs publics permettent de doubler la performance globale: ainsi, alors que les revenus provenant des entreprises privées correspondent à un indice de seulement 0,58 , les revenus obtenus d'entreprises publiques ou parapubliques permettent de hausser cet indice à 1,13 .

La performance de l'Université du Québec, enfin, se situe nettement en deçà de sa capacité virtuelle puisqu'elle est traduite par un indice de 0,76 dans le cas des revenus totaux de source corporative et de 0,68 dans le cas des revenus d'entreprises commerciales. Aucune information nouvelle ne permet de croire que la performance de l'U.Q. se soit améliorée depuis 1983. Bien au contraire, en dépit d'une population d'enseignants réguliers dont la taille relative reste constante, la part des revenus corporatifs totaux accaparée par l'Université du Québec a légèrement décliné $(-3,2 \%)$ depuis 1983-1984.

Cette rapide évaluation permet de mettre en relief une certaine division institutionnelle du financement de la recherche universitaire au Québec: d'un côté, un réseau d'universités moyennement (Montréal et sans doute Laval) ou fortement (Sherbrooke et McGill) engagées dans des programmes de collaboration avec l'entreprise (privée, publique ou parapublique); d'un autre côté, un réseau d'universités dont l'expertise est sous-utilisée par les entreprises (Université du Québec, Bishops, Concordia).

\section{Conclusion}

Ce qu'il est convenu d'appeler le "financement privé" de la recherche universitaire recouvre, en réalité, un paradoxe. En effet, une part significative du financement privé de la recherche universitaire est institutionnellement dépendante des fonds publics.

Il y a tout lieu de penser que cette tendance s'affirmera davantage au cours des prochaines années, car les gouvernements s'efforcent désormais de lier le financement public de la recherche universitaire à l'augmentation des revenus provenant des liaisons université-entreprise, soit par la création de programmes de contrepartie (Ottawa) ou par l'adoption de nouvelles formes de crédits d'impôt (Québec). Or, en vertu de ces mesures de socialisation des dépenses privées d'aide à la recherche universitaire, tout relèvement futur du soutien financier privé à la recherche universitaire sera essentiellement tributaire de l'aide gouvernementale.

Ce n'est évidemment pas un effet du hasard si la contribution des sociétés d'État au financement corporatif de la recherche universitaire, notamment de la recherche dans le réseau francophone des universités, a aujourd'hui acquis une 
telle importance quantitative. Au cours des années, les corporations publiques et parapubliques ont suppléé aux déficiences de notre système industriel.

La structure industrielle du Québec est en quelque sorte formée de deux sous-réseaux distincts. L'un est composé d'un nombre restreint de grandes entreprises concentrées dans la région montréalaise; l'autre est constitué d'un nombre élevé de PME disséminées sur une base régionale.

Ce type de structure a évidemment un impact considérable sur l'intensité et la fréquence des liaisons université-entreprise, puisque seules quelques grandes entreprises disposent d'installations ou de budget de recherche susceptibles d'appuyer les activités de recherche universitaire. Cette faiblesse du secteur manufacturier québécois se reflète notamment dans la part de ses dépenses de R-D en proportion de la valeur ajoutée industrielle. En 1984, par exemple, le rapport $R-D_{i} / V_{i}$ du Québec pour l'ensemble du secteur manufacturier $(2,19 \%)$, était non seulement inférieur à celui de l'Ontario $(2,93 \%)$, mais également à la moyenne nationale $(2,47 \%)$. Dans le secteur des industries de haute technologie, la performance québécoise s'avérait plus décevante encore: $13,2 \%$ au Québec, comparativement à 15,6\% au Canada et 20,0\% en Ontario (Leclerc, 1987).

Il n'est pas étonnant de constater, dans ce contexte, que les dépenses consenties par les industries du Québec en 1985 ne totalisent que 24,0\% de l'effort canadien de la R-D industrielle, tandis que les industries ontariennes absorbent $59,0 \%$ des dépenses canadiennes totales à ce chapitre. Cet écart se répercute également au niveau de la collaboration université-entreprise. Les entreprises commerciales de l'Ontario supportent en effet $55,0 \%$ des revenus universitaires canadiens de recherche provenant du secteur industriel, alors que les industries du Québec sont responsables de 33,3\% seulement de ces revenus (Statistique Canada, 1987).

Mais les caractéristiques de la structure industrielle, pour déterminantes qu'elles soient, ne sont pas seules en cause lorsqu'il s'agit d'identifier les incitations au financement privé de la recherche universitaire. Une enquête de l'OCDE (1984, p. 20) suggère en effet que le soutien du secteur privé à l'effort de recherche d'une université est en relation directe avec la qualité de l'enseignement et de la recherche dans cette université. En d'autres termes, le niveau du financement privé constituerait un indice de l'excellence universitaire. En revanche, il existerait peu de rapports entre la qualité scientifique de la recherche et son adaptation aux exigences de l'industrie (Blume et Sinclair, 1973). Mais c'est là un autre paradoxe du financement privé de la recherche universitaire sur lequel il faudra bien un jour tenter de réfléchir.

\section{Notes}

1 L'expression "contribution directe" désigne les revenus versés aux universités sous forme de commandite, contrat ou subvention, à l'exclusion des legs, des dons, des revenus d'investissements ou de tout autre revenu de source privée (prêt d'équipement ou de personnel, soutien technique, échange d'informations, etc.).

2 Les indices de performance normalisent les revenus de recherche de source corporative sur la base de la répartition provinciale des professeurs-chercheurs (professeurs à plein temps). Chacun de ces 
indices est fondé sur l'hypothèse selon laquelle, toutes choses étant égales par ailleurs, chaque université devrait obtenir une part des revenus-réseau de source corporative proportionnelle à l'importance numérique relative de ses effectifs. Cette équivalence statistique est traduite par le coefficient 1,00. En conséquence, tout coefficient supérieur à l'unité indique qu'une université a obtenu une proportion des revenus-réseau de source corporative (privée et/ou publique) supérieure à sa quote-part théorique.

\section{RÉFÉRENCES}

Advisory Board for the Research Councils (1984). Scientific Opportunities and the Research Budget: A Report to the Secretary of State, Londres.

Ashworth, J. M. (1985). "What price an ivory tower? University-industry relationship", Higher Education Review, printemps, 17 (2), 31-43.

Blume, S.S. et Sinclair, R. (1973). "Chemists in British Universities: a Study in the Reward System of Science", American Sociological Review, 38, février, 126-138.

Blume, S.S. (1985). "After the Darkest Hour: Integrity and Engagement in the Development of University Research", in Wittsock, B. et Elzinga, A. (éds.), The University Research System, Stockholm.

Blume, S.S. (1984). "La politique scientifique à l'université et ses conséquences pour la planification et l'administration", Revue internationale de gestion des établissements d'enseignement supérieur, juillet, 8(2), 189-202.

Chapdelaine, B. (1987). "Le mariage Universités-Entreprises: les PME disent NON", Hebdo-science, 22 octobre.

Conseil de la science et de la technologie (1986). La collaboration université-entreprise et le financement de la recherche universitaire, Quebec.

Conseil de la science et de la technologie (1987). Bilan des activités scientifiques et techniques de la région de Québec (03), Quebec.

Dickson, D. (1984). The New Politics of science, New York, Pantheon Books.

Kennedy, D. (1986). "Basic Research in the Universities: How much "Utility", in Landau, R. et Rosenberg, N. (éds.), The Positive Sum Strategy, Washington, D.C., National Academy Press, 263-2744.

Leclerc, M. (1987). Indicateurs des flux technologiques internationaux: Québec, Ontario et Canada, 1981-1985, Rapport présenté au Conseil de la science et de la technologie du Québec, le 27 mars 1987, 136 pages.

Lemasson, J.P. (1985). Les relations universités-industries au Québec. Pour des nouvelles voies d'analyse, Congrès des sociétés savantes, Université de Montréal, mai, 15 pages.

Mansfield, E. (1981). "Composition of R-D expenditures: relationship to size of firm, concentration and innovative input", Review of Economics and Statistics, LXIII (4), novembre, 610-615.

Ocde (1984). Industrie et université, Paris.

Ocde (1987a). La contribution de la science et de la technologie au développement économique et social. Perspectives et conditions, SPT (87) 14, Paris.

Ocde (1987b). Quel avenir pour les universités?, Paris.

Philippart, A. (1985). "Stimulations et entraves à la recherche et au développement”, in Fuks, R. (éd.), La recherche universitaire, la recherche industrielle, les pouvoirs publics, une collaboration possible, Bruxelles, Éditions de l'université de Bruxelles, 175-216.

Ruscio, K.P. (1984). "The Changing Context of Academic Science: University Industry Relations in Biotechnology and the Public Policy Implications", Policy Studies Review, novembre 4 (2), 259-275.

Statistique Canada (1987). Les estimations des dépenses canadiennes au titre de la recherche et du developpement, par région, 1979 à 1985, Ottawa. 